INRA Prod. Anim.,

2012, 25 (4), 375-388

\title{
Organisation des filières animales et environnement. Vingt ans après la directive nitrates
}

\author{
C. GAIGNÉ $1,2,3$ \\ ${ }^{1}$ INRA, UMR1302 Structures et Marchés Agricoles, Ressources et Territoires, \\ 4 allée Adolphe Bobierre, F-35000 Rennes, France \\ 2 Agrocampus Ouest, UMR Structures et Marchés Agricoles, Ressources et Territoires, \\ 65 rue de Saint-Brieuc, F-35000 Rennes \\ ${ }^{3}$ CREATE, Université de Laval, Québec, Canada \\ Courriel : carl.gaigne@rennes.inra.fr
}

Face aux puissants mécanismes de marché poussant à la concentration spatiale des filières animales, les mesures environnementales peinent à réduire les excès d'azote dans les territoires à forte densité animale. La montée du prix de l'énergie et la dérèglementation des marchés agricoles ne seront pas sans conséquences sur les niveaux de pollution selon les territoires. La régulation environnementale pourrait être plus efficace si elle ciblait également les industriels de ces filières animales.

Depuis plusieurs décennies, on assiste en France à un mouvement de concentration de la production animale au niveau des régions et des exploitations. Les effectifs des différents cheptels se concentrent à la fois dans quelques bassins de production et dans les grandes exploitations. Cette dynamique d'agglomération s'accompagne d'un processus de concentration industrielle et spatiale dans les secteurs se situant à l'aval et à l'amont des élevages, c'est-à-dire les secteurs de la viande, du lait et de la fabrication d'aliments pour animaux de ferme. Ce double mouvement de concentration industrielle et spatiale caractérisant les filières animales en France est observé dans de nombreux pays d'Europe et d'Amérique du Nord (Abdalla et al 1995). Cette agglomération des productions animales se traduit cependant par une concentration des effluents d'élevage, dont l'épandage a des conséquences environnementales souvent néfastes. C'est pourquoi certaines mesures de régulation environnementale ont vu le jour dans différents pays, notamment au travers de la directive «Nitrates» de 1991 (Conseil des communautés européennes 1991). Vingt ans après cette mesure de l'Union Européenne, cet article questionne la capa- cité des régulations environnementales liées à la gestion des effluents à déconcentrer les productions animales et à réduire les excès d'azote au niveau des territoires à forte densité de cheptel.

Nous puiserons tout d'abord dans la théorie économique et les données disponibles pour mettre en évidence les mécanismes à l'origine de la forte agglomération des productions animales et de ses industries ${ }^{1}$. La localisation des activités et la spécialisation des territoires ne sont pas le fruit du hasard. Nous mettrons en évidence que la tendance à la baisse du prix relatif de l'énergie depuis plusieurs décennies et la présence d'économies d'échelle aux différents stades des filières animales peuvent constituer des facteurs importants de ce processus de concentration des productions animales. D'une part, la baisse des prix relatifs des engrais chimiques et des coûts de transport des céréales a créé les conditions pour que l'élevage soit chassé des meilleures terres au profit des grandes cultures. D'autre part, la réalisation d'économies d'échelle favorisant l'agrandissement des exploitations se ferait, dans l'élevage, au détriment des économies de gamme (qui favorisent l'association de différentes productions dans une même unité de production). Mais cela ne suffit pas à expliquer la concentration géographique au niveau de quelques bassins de production : on peut avoir des exploitations spécialisées dispersées.

La réforme des politiques agricoles, tendant notamment vers un découplage des aides, a également réduit la dispersion des productions agricoles et a permis l'émergence de deux facteurs, moins connus dans le champ de l'économie agricole, contribuant à cette concentration spatiale des productions animales. Il s'agit tout d'abord des gains économiques liés à la concentration spatiale, que l'on nomme désormais économies d'agglomération, comme l'a montré Paul Krugman, prix Nobel d'Economie (Krugman 1997). De nombreux travaux empiriques mettent en évidence une relation positive forte entre la taille économique d'un territoire et son niveau de productivité (Rosenthal et Strange 2004). Nous reviendrons plus loin sur la nature de ces économies d'agglomération. Cette relation positive entre degré de concentration spatiale et niveau de productivité se vérifie également pour les activités agricoles. L'histoire des Etats-Unis est intéressante de ce point de

\footnotetext{
${ }^{1}$ La concentration de la production agricole et la spécialisation des territoires a fait l'objet de nombreux travaux depuis la révolution industrielle (se reporter par exemple à l'ouvrage collectif «Histoire de la France rurale sous la direction de Duby et Wallon»). Dans ce qui suit, je discuterai les éléments factuels à la lumière des théories économiques récentes et positives, mettant en avant des facteurs économiques des dynamiques observées. Evidemment, d'autres dimensions existent comme les éléments culturels, sociologiques, etc. Pour la clarté de notre exposé et dans un souci de cohérence, l'accent sera mis sur la dimension économique - les mécanismes de marchés et les régulations économiques.
} 
Tableau 1. Evolution de la taille moyenne des élevages.

\begin{tabular}{|l|c|c|c|c|c|c|}
\hline & \multicolumn{2}{|c|}{ Nb. Exploitations } & \multicolumn{2}{c|}{ Effectif des cheptels } & \multicolumn{2}{c|}{ Taille moyenne } \\
\hline Type de cheptel & 2000 & 2010 & 2000 & 2010 & 2000 & 2010 \\
\hline Vaches & 270635 & 185989 & 8558746 & 7856506 & 32 & 42 \\
\hline Bovins & 296604 & 199624 & 20388920 & 19506209 & 69 & 98 \\
\hline Porcins & 67403 & 24454 & 15001728 & 13921515 & 223 & 569 \\
\hline Volailles* & 240422 & 76319 & 78253155 & 78062423 & 325 & 1023 \\
\hline
\end{tabular}

*Poules et poulettes.

Source : Agreste, Recensement Agricole 2000 et 2010.

vue. En effet, dans son célèbre ouvrage, Nature's Metropolis, l'historien William Cronon propose une description détaillée de la concentration des productions animales américaines au XIX ${ }^{\text {ème }}$ siècle dans la région de Chicago (Cronon 1991) (voir en particulier son chapitre judicieusement intitulé Porkopolis). Il $\mathrm{y}$ insiste sur le rôle fondamental qu'a joué dans ces filières la proximité géographique, entre d'une part les éleveurs et d'autre part les industries de grande dimension développées dans cette ville. Le second facteur, moins évident, concerne les évolutions des industries des filières animales qui ont des implications spatiales non négligeables. Nous verrons de quelle manière les restructurations industrielles dues à l'intégration verticale ont favorisé la spécialisation régionale dans les productions animales.

Les effets environnementaux indésirables liés à cette concentration spatiale des productions animales sont aujourd'hui indéniables et sont de plus en plus dénoncés. Ainsi, à l'occasion du Grenelle de l'Environnement en 2007, la question de la localisation des productions agricoles et notamment de leur dispersion a été évoquée comme un levier possible pour réduire les problèmes environnementaux. Sans avoir attendu ce débat, l'Union Européenne a, au travers de la directive «Nitrates», cherché à limiter la pollution des eaux liée à une trop forte concentration en nitrates. L'épandage des effluents est limité par un niveau maximum d'azote par hectare et par an. Cette restriction des droits d'épandage a logiquement engendré des difficultés dans les régions à forte densité de cheptel et où les surfaces disponibles ou accessibles pour l'épandage n'étaient pas suffisantes. La mise en œuvre de cette directive se traduit, non seulement par des coûts non négligeables notamment de mise aux normes, de transport ou de traitement des effluents, mais aussi par la création de liens entre les productions dites hors-sol et la terre. Elle peut ainsi potentiellement jouer le rôle de force de dispersion de la production en limitant le développement des élevages dans les zones de forte densité animale. Nous verrons dans cet article dans quelle mesure les différents instruments de régulation environnementale mis en œuvre ont eu des effets limités sur la réduction des excès d'azote dans certains territoires.

\section{1 / Origines économiques des excès d'azote au niveau des territoires}

Les origines économiques de la concentration spatiale des productions animales sont diverses. Les exploitations et les territoires se sont tout d'abord de plus en plus spécialisés dans les productions animales ou végétales. Deux événements en sont à l'origine qui ont pour cause commune la baisse des prix de l'énergie. Le premier événement concerne la baisse relative des prix des fertilisants chimiques dans les années 1980 et 1990 (GCL Développement Durable 2010). Cette baisse a favorisé l'usage croissant de fertilisants chimiques au détriment de l'azote organique. Le second événement est relatif à la baisse des coûts et temps de transport qui a permis d'élargir les marchés des débouchés et de l'approvisionnement. Les produits relativement légers et à forte valeur ajoutée (cas des engrais minéraux, des grains, des produits de substitution aux céréales, des tourteaux protéagineux et des produits alimentaires) ont voyagé sur des distances de plus en plus grandes. En raison de ces deux événements, les régions dotées de terres fertiles se sont spécialisées dans les productions végétales pour fournir de manière croissante les marchés extérieurs, en écartant progressivement les activités d'élevage. En revanche, les productions animales se caractérisent notamment par des produits relativement lourds et à faibles valeurs ajoutées et elles se sont donc concentrées autour des établissements portuaires ou industriels d'amont (productions hors-sol près des ports) ou d'aval (lait près des laiteries). Ainsi, le commerce interrégional et international de céréales, de produits de substitution aux céréales et aux protéagineux destinés à l'alimentation animale (entrant sans droit de douane en Europe dès le Kennedy round 1967) s'est développé. Evidemment, d'autres facteurs ont également contribué à ce processus de division spatiale du travail et de spécialisation des territoires, que nous détaillons dans ce qui suit.

\section{1 / Économies de coût liées à la taille et à la spécialisation des élevages}

La présence d'économies liées à la taille des exploitations, signifiant qu'un accroissement de la production se traduit par une baisse du coût moyen de production, provoque la spécialisation des exploitations. De telles économies sont suggérées par le constat concernant les évolutions parallèles de la productivité et de la spécialisation des élevages. Cela est une caractéristique technologique, encouragée par les rapports de prix, en particulier la croissance plus rapide du prix du travail par rapport à ceux des autres facteurs, qui conduit à la spécialisation des exploitations (Dupraz 1997).

La hausse continue de la productivité globale des facteurs dans les exploitations dédiées aux productions animales est observée depuis plusieurs décennies (même si cette hausse diminue ces dernières années) en France comme dans de nombreux pays (Butault 2006). Par ailleurs, la taille moyenne des exploitations a encore significativement augmenté depuis 2000 (tableau 1) pour les effectifs des cheptels bovins et vaches. Cette hausse de la taille moyenne atteint des proportions encore plus importantes pour la volaille et le secteur porcin. Non seulement la taille moyenne des exploitations augmente, mais la production se concentre de plus en plus dans les élevages de grande taille quel que soit le type de cheptel. Les exploitations porcines avec plus de 200 truies concentrent près de la moitié des effectifs de truies (tableau 2).

Ce mouvement de concentration de la production s'accompagne d'un renforcement de la spécialisation des exploitations (Desrier 2011). Depuis 1970, on est passé grossièrement d'une situation où le secteur agricole était dominé par les exploitations mixtes à une situation 
Tableau 2. Evolution du poids des plus grandes exploitations en termes de taille des cheptels.

\begin{tabular}{|c|c|c|c|}
\hline Type de cheptel & & $\mathbf{2 0 0 0}$ & $\mathbf{2 0 1 0}$ \\
\hline Vaches & Effectif de la tranche & 1426565 & 2569817 \\
80 têtes et plus & Part de la tranche & $17 \%$ & $33 \%$ \\
\hline Bovins & Effectif de la tranche & 4230347 & 7034317 \\
\hline Truies mères & Part de la tranche & $21 \%$ & $36 \%$ \\
200 têtes et plus & Effectif de la tranche & 623510 & 611414 \\
\hline Porcs à l'engraissement & Part de la tranche & $43 \%$ & $54 \%$ \\
1500 têtes et plus & Effectif de la tranche & 2140276 & 2812100 \\
\hline Volaille** & Part de la tranche & $28 \%$ & $33 \%$ \\
50 milliers de têtes et plus & Effectif de la tranche & 39102798 & 47012406 \\
\hline
\end{tabular}

*1200 têtes et plus pour l'année 2000 ; **Poules et poulettes.

Source : Agreste, Recensement Agricoles 2000 et 2010.

où les exploitations spécialisées, animales et végétales, fournissent l'essentiel des productions au détriment des exploitations mixtes ${ }^{2}$. En effet, les facteurs de production, comme le travail, qui sont économisés par l'agrandissement d'un atelier voient leur prix unitaire croître plus rapidement que ceux, comme l'énergie et les engrais minéraux, qui sont économisés par l'association des productions animales et végétales. Dupraz (1997) montre empiriquement que l'agrandissement d'un atelier de production et l'association des productions ne vont pas de pair. La raison en est sans doute la spécificité des compétences pour chaque production et les coûts d'organisation internes liés à l'association de ces compétences. Par ailleurs, l'usage croissant de pesticides et d'antibiotiques a favorisé la spécialisation des exploitations en permettant la simplification des assolements et la concentration des élevages.

Le double processus de concentration de la production et de spécialisation des exploitations reflète les caractéristiques des technologies agricoles mises en œuvre. Il s'agirait d'une part, de l'existence d'économies d'échelle - l'accroissement de la production d'un bien s'accompagne d'une diminution des coûts moyens de production - et d'autre part, de la faiblesse relative des économies de gamme réalisées par l'association de différents types de productions au sein d'une même entreprise - la baisse des coûts due à la production conjointe par rapport à la production séparée de quan- tités égales de biens. Le mouvement de spécialisation des exploitations suggère que les économies de gamme sont insuffisantes, voire négatives dans certains cas, pour compenser les économies d'échelle réalisées en monoproduction.

Au niveau de l'exploitation, les économies de gamme peuvent provenir des engrais minéraux économisés en raison de l'association agriculture et élevage permettant l'utilisation fertilisante des effluents animaux ou, éventuellement, d'une baisse des coûts d'achat de l'aliment industriel due à la fabrication à la ferme de l'alimentation pour les animaux. Les facteurs de production contribuant aux économies d'échelle sont généralement le travail. Par ailleurs, certains équipements caractérisant la production spécialisée, comme les installations d'élevage, génèrent des coûts fixes non récupérables et donc sont plus aisément amortis si on produit à grande échelle. Les travaux empiriques descriptifs montrent l'existence d'une relation en «L» entre coût moyen de production et niveau de production (Chavas 2008). Autrement dit, le coût moyen diminue quand la production augmente pour les exploitations de petite taille et le coût moyen évolue peu avec la production à partir d'une certaine taille. Ceci a deux implications. Tout d'abord, l'absence de «déséconomies» d'échelle au niveau de la production agricole (les coûts moyens de production n'augmenteraient pas en moyenne avec le niveau de la production). Ensuite, il existe une taille minimum pour être effi- cace. Toutefois, à ce niveau d'analyse, on compare des exploitations de grande taille et de petite taille qui peuvent utiliser des technologies différentes. Autrement dit, peuvent coexister des technologies à rendements croissants puis décroissants à partir d'un certain seuil de production, mobilisées par les petites exploitations et des technologies à rendements constants utilisées par les grandes exploitations.

Les économies de coût liées à la taille des exploitations ne s'expliquent pas uniquement par des facteurs liés à la technologie de production. Les grandes exploitations peuvent payer moins cher certains biens intermédiaires en raison d'une part, de la présence de coûts de transaction et de transport et, d'autre part, d'un plus grand pouvoir de négociation. C'est le cas par exemple de l'alimentation industrielle pour les porcs. Les fournisseurs peuvent réaliser des économies lorsqu'ils livrent un éleveur de grande taille plutôt que plusieurs petits éleveurs. Ces gains monétaires sont capitalisés dans le prix du bien intermédiaire. C'est pourquoi le prix d'achat des aliments est négativement corrélé avec la taille des exploitations porcines (Duvaleix-Tréguer et Gaigné 2011). En revanche, pour certains facteurs de production comme le travail, les coûts de transaction augmentent avec la taille. En effet, le coût de la gestion de la maind'œuvre augmente avec le nombre de salariés (Chavas 2008). Dans ce cas, la grande taille des exploitations peut impliquer des désavantages ${ }^{3}$.

\footnotetext{
2 On observe des évolutions comparables aux Etats-Unis. Par exemple, le nombre de fermes ayant des vaches laitières a diminué de façon constante et fortement entre 1970 et 2006 tandis que la taille moyenne des troupeaux est passée de seulement 19 vaches par ferme en 1970 à 120 vaches en 2006 (MacDonald et McBride 2009). En outre, parce que la production de lait par vache a doublé entre 1970 et 2006, la production moyenne de lait par exploitation a été multipliée par douze. Le nombre de fermes de plus de 2000 vaches a doublé entre 2000 et 2006, et celles-ci représentent $25 \%$ de la production de lait. Concernant le secteur bovin à viande, en 2007, 262 exploitations bovines ont une capacité d'au moins 16000 têtes et assurent à elles seules $60 \%$ des mises en marché des bovins produits aux Etats-Unis (la plus grande peut nourrir 100000 bovins).

3 Il existe par ailleurs un lien entre l'exposition aux risques (de prix et de production) et la taille des exploitations. D'une part, l'exposition au risque est plus forte pour les exploitations de grande taille. D'autre part, la grande taille permet une meilleure gestion du risque. Néanmoins, nous manquons de travaux empiriques pour déterminer quel est l'effet dominant.
} 
Le recours à des outils d'inférences statistiques est nécessaire pour déterminer l'existence et l'ampleur des économies de coûts liés à la taille des exploitations. De manière générale, les travaux économétriques montrent que les économies de coûts liées à la taille des exploitations ne sont pas négligeables dans les élevages laitiers Européens et d'Amérique du Nord tandis que les économies de gamme sont faibles (Wieck et Heckelei 2007, Mosheim et Lovell 2009). Dans le secteur du porc, on note cependant la présence d'économies d'échelle d'après des données américaines (Azzam et Skinner 2007) et des données françaises (DuvaleixTréguer et Gaigné 2011).

\section{2 / Économies liées à la con- centration spatiale de la pro- duction}

La hausse de la productivité des exploitations spécialisées dans les productions animales peut également être associée à l'accroissement de la concentration spatiale des productions animales. En effet, ce secteur d'activité de l'agriculture est particulièrement concentré géographiquement, comme l'illustre la figure 1 représentant la répartition spatiale des rejets d'azote organique par les animaux. Les productions animales sont en moyenne plus concentrées spatialement que les productions végétales (Ben Arfa et al 2009). Par ailleurs, les productions animales non soutenues directement par la PAC (secteurs porcins et avicoles) sont davantage concentrées géographiquement que les autres productions. Dans le secteur bovin, la gestion des quotas a été un instrument pour figer régionalement l'offre de lait et par conséquent un frein à la concentration. La prime à la vache allaitante (PMTVA, seule aide restée couplée) ainsi que les aides pour le maintien des surfaces en herbe et pour compenser les handicaps naturels ont soutenu la présence des ruminants dans des zones difficiles.

Toutefois, quel que soit le secteur d'activité (hors-sol, ruminants) l'agglomération des productions animales semble donc être une source de gains de productivité et/ou d'économies d'échelle. On sait depuis longtemps que la manière dont les activités économiques sont réparties dans l'espace n'est pas neutre sur la performance économique des producteurs. Les nombreux travaux relevant de l'économie géographique ont bien mis en évidence les gains économiques liés à la concentration géographique des

Figure 1. Répartition spatiale des rejets d'azote organique (kg/ha de surface épandable) en 2010.

Source : Carto Dynamique INRA.

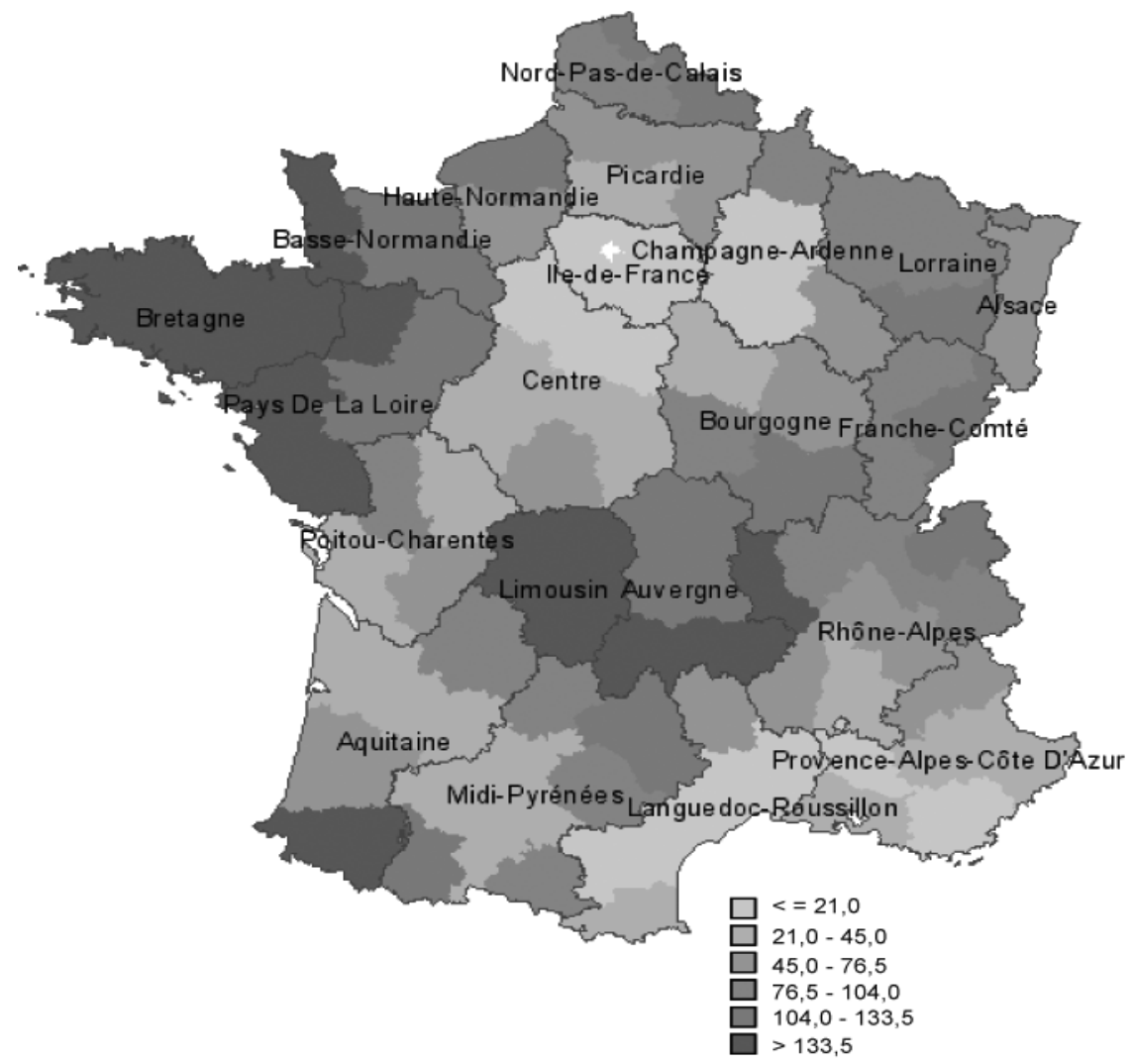

entreprises (Krugman 1997). Le message de Paul Krugman est simple : même sans avantage comparatif, une région peut se spécialiser dans un secteur d'activité. Il soutient que, depuis la révolution industrielle, le développement économique résulte de la combinaison des économies d'échelle (plus on produit, moins cela coûte par unité produite) et des coûts de transport. Sans nier le rôle des ressources disponibles, l'existence de gains tirés de la concentration géographique de la production devient un élément central pour expliquer la spécialisation des régions ${ }^{4}$.

La littérature montre également que les producteurs peuvent aussi bénéficier de la simple proximité géographique entre producteurs d'un même secteur. Les interactions non marchandes, qu'ils nouent d'autant plus facilement entre eux qu'ils sont proches, permettent de modifier la relation entre coût et production. La proximité géographique entre producteurs favorise la circulation rapide et fiable (ou le partage) d'informations relatives aux évolutions des marchés de fournitures ou de produits, mais aussi au développement d'innovations techniques, organisationnelles ou de produits (Fujita et Thisse 2002). Nous avons alors affaire à ce qui est communément nommé «externalités» d'information. La proximité permet également aux producteurs de partager des équipements communs dont les investissements ne seraient, au regard de l'usage qu'ils en ont, pas supportables individuellement, ou dans certains cas, une main-d'œuvre formée aux tâches spécifiques de cette production. La fréquence des contacts permet en outre aux clients et aux fournisseurs de construire des relations de confiance nécessaires à la rédaction de contrats. Dit autrement, l'efficacité productive des élevages s'accroît avec le nombre d'élevages localisés dans la même zone géographique et s'atténue à mesure qu'augmente la distance entre eux. Tous ces facteurs non marchands favorisant l'agglomération relèvent de ce que l'on nomme en économie spatiale les «externalités technologiques ou marshalliennes».

Ces externalités spatiales positives seraient en œuvre dans les élevages. Cinq études ont adopté la même méthodologie pour tester la présence d'économies d'agglomération. Il s'agit

\footnotetext{
4 Evidemment, la faible quantité de terre par actif, par exemple en Bretagne, participe aussi à l'émergence d'une production intensive. Toutefois, il faut être très prudent sur la capacité prédictive de la théorie sous-jacente dite des avantages comparatifs.
} 
de trois études sur la filière porcine, à partir de données danoises (Larue et al 2011), françaises (Gaigné et al 2011) et des Etats-Unis (Roe et al 2002) et de deux études sur la filière lait à partir de données françaises (Ben Arfa et al 2010) et des Etats-Unis (Isik 2004). Ces cinq études concluent que les filières animales sont caractérisées par des externalités spatiales positives liées à la proximité entre éleveurs. Autrement dit, la proximité géographique entre éleveurs leur permet d'accroître leur productivité. Ce résultat demeure même si l'on contrôle la relation causale inverse (une hausse de la productivité accroît la concentration spatiale de la production) et la présence de facteurs de localisation communs (proximité des industries d'abattage et d'alimentation animale notamment). Notons également que les économies d'agglomération sont particulièrement importantes dans le secteur porcin. En effet, si on a observé une délocalisation des productions porcines aux Etats-Unis à la fin du $\mathrm{XX}^{\text {ème }}$ siècle, celle-ci n'a pas débouché sur une plus grande dispersion entre les Etats américains, mais sur une forte concentration spatiale dans d'autres Etats (Hubbell et Welsh 1998).

Les changements opérés au niveau des exploitations agricoles ne sont pas suffisants pour expliquer l'ampleur de la concentration spatiale des productions animales. Les évolutions dans les industries d'amont et d'aval ont également participé à ce mouvement de concentration des activités d'élevages.

\section{3 / Des économies d'échelle permises par la concentration spatiale dans les industries d'amont et d'aval}

Quelle que soit l'industrie liée aux productions animales, on assiste à un mouvement de concentration de l'appareil productif. Dans les quatre grandes industries (de la viande de boucherie et de volaille, du lait, de produits à base de viande, de la fabrication d'aliments pour le bétail), le nombre d'entreprises a baissé, la taille moyenne des entreprises a augmenté ainsi que la taille des plus grandes entreprises et leur productivité, comme le montre le tableau 3. Ceci est observé dans différents pays industrialisés. Différents travaux économétriques mettent en évidence la présence d'économies d'échelle dans les établissements et les entreprises des industries d'aval (MacDonald et Ollinger 2000, Morrison Paul et al 2001, Ollinger et al 2005, Gervais et al 2008). Le corollaire de cette concentration industrielle est une plus grande concentration spatiale de la production animale par le jeu de fermeture d'établissements et l'agrandissement de certaines usines.

Ce mouvement de concentration de la production dans les industries en aval et en amont devrait vraisemblablement se poursuivre en France, quel que soit le type d'activité. En effet, les changements en cours dans les différentes activités mènent vers une plus grande concentration industrielle des activités se situant à l'aval et à l'amont des élevages. Par exemple, dans l'industrie de la viande de volaille, les quatre premiers groupes assuraient $63 \%$ du chiffre d'affaires de la filière volailles de chair produites en France en 2007, et les dix premiers $80 \%$. Néanmoins, la concentration dans le secteur avicole demeure plus faible en France que dans d'autres pays, notamment européens et il existe de nombreuses petites et moyennes entreprises. Le processus de concentration sectorielle devrait donc se poursuivre dans les prochaines années.

La concentration devrait se poursuivre aussi dans l'industrie de la viande de boucherie. En effet, si la tendance à la baisse sur le long terme de la consommation de viande fraîche est relativement stable, la production et la consommation de produits élaborés ont fortement progressé depuis 2001 (de près de 50\% entre 2001 et 2007). Par ailleurs, malgré la diminution du nombre d'abattoirs, ceux-ci demeurent en surcapacité.

De même, l'industrie du lait compte près de 300 entreprises de plus de 20 salariés, mais 10 groupes seulement transforment $75 \%$ du lait produit en France. Ces dix groupes transforment chacun au minimum plus de 500 millions de litres de lait. Ces restructurations prennent la forme d'acquisitions pour accroître la gamme de produits ou pour exploiter les économies d'échelle au niveau des établissements et accroître le volume de lait disponible. Des partenariats sont également en œuvre pour accroître les parts de marché ou les pouvoirs de négociations avec les acteurs de l'aval.

Tableau 3. Dynamique des industries des viandes et de l'alimentation animale.

\begin{tabular}{|l|l|c|c|c|}
\hline \multicolumn{1}{|c|}{ Industrie } & & $\mathbf{1 9 9 6}$ & $\mathbf{2 0 0 0}$ & $\mathbf{2 0 0 7}$ \\
\hline \multirow{5}{*}{ Viande de boucherie } & Nombre d'entreprises [1] & 460 & 429 & 367 \\
& Taille moyenne (effectif/[1]) & 91 & 110 & 130 \\
& Effectif de la plus grande entreprise & 1890 & 2425 & 4501 \\
& Productivité travail (Val. Ajoutée/effectif) & 33,11 & 34,48 & 42,89 \\
\hline \multirow{5}{*}{ Viande de volaille } & Nombre d'entreprises [1] & 199 & 186 & 160 \\
& Taille moyenne (effectif/[1]) & 135 & 155 & 181 \\
& Effectif de la plus grande entreprise & 2042 & 1749 & 2797 \\
& Productivité travail (Val. Ajoutée/effectif) & 34,28 & 35,13 & 39,40 \\
\hline \multirow{5}{*}{ Produits à base } & Nombre d'entreprises [1] & 462 & 437 & 383 \\
de viande & Taille moyenne (effectif/[1]) & 91 & 106 & 112 \\
& Effectif de la plus grande entreprise & 1329 & 1457 & 1561 \\
\hline \multirow{5}{*}{ Industrie du lait } & Productivité travail (Val. Ajoutée/effectif) & 33,55 & 40,66 & 44,40 \\
& Nombre d'entreprises [1] & 344 & 312 & 294 \\
& Taille moyenne (effectif/[1]) & 170 & 190 & 181 \\
\hline \multirow{3}{*}{ Fabrication d'aliments } & Effectif de la plus grande entreprise & 3347 & 3155 & 2394 \\
pour le bétail & Productivité travail (Val. Ajoutée/effectif) & 40,51 & 41,97 & 61,92 \\
\hline & Nombre d'entreprises [1] & 220 & 215 & 174 \\
& Taille moyenne (effectif/[1]) & 58 & 61 & 65 \\
& Effectif de la plus grande entreprise & 449 & 531 & 813 \\
\hline
\end{tabular}

${ }^{*}$ Effectif $=$ nombre de salariés.

Source : Enquête Annuelle d'Entreprises (Valeur Ajoutée en million d'euros). 
L'industrie de préparation industrielle de produits à base de viande, qui transforme $70 \%$ de la production porcine française, est également dominée par des entreprises multinationales. Comme pour les autres secteurs liés aux productions animales, la tendance est à la concentration des entreprises. Ce mouvement de concentration risque de se poursuivre afin de faire face à la concentration dans le secteur de la grande distribution (plus de $80 \%$ de la production de charcuterie est vendue en grandes et moyennes surfaces).

L'industrie de la fabrication d'aliments pour animaux en France est composée, en 2007 , de 210 entreprises de fabrication, dont 174 de plus de 20 salariés et 301 sites. Ces chiffres sont en baisse constante depuis plus de vingt ans; le nombre d'entreprises (par rachat et cessation d'activité) et de sites (par fermeture) a été divisé par deux depuis 1988 . La taille moyenne des entreprises et des établissements augmente constamment depuis vingt ans. Aujourd'hui, 29 entreprises assurent $62 \%$ de la production.

\section{4 / Rôle de la proximité géo- graphique des éleveurs et des industriels}

Les relations entre l'agriculture et les industries sont également un facteur puissant d'agglomération des différentes étapes des filières agro-alimentaires (Bagoulla et al 2010). L'agglomération de la production est un processus de long terme pour partie dû aux gains qui existent pour les firmes à se localiser à proximité de leurs clients (industries d'aval) et de leurs fournisseurs (industries d'amont) pour bénéficier d'économies d'échelle et de coûts de transport bas. L'idée est simple : les producteurs sont incités à se localiser près de leurs clients pour diminuer leurs prix, grâce à de moindres coûts de transport des marchandises. Cette baisse suscite une demande plus élevée et permet donc de produire à plus grande échelle. Accrô̂tre le niveau de production fait baisser les coûts moyens en raison des économies d'échelle. Production en hausse et coûts moyens de production en baisse permettent d'accroître les niveaux de profits. De même, pour éviter les coûts monétaires et les pertes de temps liés à l'éloignement géographique, les entreprises sont incitées à se rapprocher de leurs fournisseurs. Le regroupement géographique permet de réduire le prix des biens intermédiaires, les délais de livraison du bien ou du service et d'obtenir plus facilement les caractéristiques précises du produit souhaité. Les firmes appartenant à une même filière s'attirent donc spontanément. Les travaux de Roe et al (2002), Isik (2004) et Gaigné et al (2011) suggèrent que de tels mécanismes sont en œuvre dans les filières du porc et du lait.
Ces mécanismes évoqués précédemment expliquent assez bien la spécialisation de la Bretagne dans les productions animales. L'industrie de production de viande de boucherie s'y est fortement développée au cours des dernières décennies. La région est le premier bassin d'emploi de ce secteur, avec environ 14000 emplois salariés. Ce n'est pas un hasard si, en Bretagne, cette industrie s'est surtout développée dans la zone d'emploi de Saint-Brieuc (un emploi sur quatre), là où la production animale est très concentrée. En dépit de la baisse des coûts de transport en Bretagne, l'activité d'abattage et de découpe s'est encore rapprochée des lieux d'élevage. Cette décroissance des coûts de transport a permis d'exploiter les économies d'échelle dans cette activité industrielle. Les gains de productivité qui en découlent, à ce stade de la production, ont permis en retour d'accroître la production animale. Ceci explique également pourquoi la Bretagne reste la première région en termes de préparation industrielle de produits à base de viande (9000 emplois), mais aussi pour la fabrication d'aliments pour animaux de ferme (4000 emplois). Aujourd'hui, l'industrie agroalimentaire est le premier secteur exportateur en Bretagne (plus de 2 milliards d'euros), bien loin devant l'industrie automobile et l'industrie des biens d'équipements.

\section{5 / Rôle de l'intégration verti- cale}

Abordons un dernier facteur explicatif de la concentration spatiale des productions animales peu évoqué malgré son importance : l'intégration verticale au sein des filières animales. On a assisté, ces deux dernières décennies non seulement à une multiplication des acquisitions et fusions d'entreprises agroalimentaires, mais aussi au renforcement de l'intégration verticale se traduisant par un plus fort contrôle des industriels sur les fournisseurs des produits agricoles. En réduisant le nombre de décideurs et en relâchant la concurrence entre industriels, le niveau de décision est devenu ainsi très centralisé. La gestion des risques liés aux variations de prix ou de production par les choix de production, est de moins en moins réalisée au niveau de l'exploitation mais de plus en plus au niveau des industriels de l'aval. Autrement dit, les exploitants peuvent moins diversifier leurs activités pour réduire le risque de pertes de revenu contrairement aux industriels. Par exemple, les producteurs de lait sont en moyenne vulnérables aux aléas de prix comme l'ont montré les années 2008 et 2009. Par ailleurs, les industriels sont incités d'une part, à réduire le nombre de fournisseurs pour minimiser les coûts de transaction et, d'autre part, à favoriser la spécialisation des élevages pour exploiter les économies d'échelle. Ainsi, les industriels peuvent gérer les risques en se dotant d'un portefeuille diversifié d'activités constituées d'unités de production avec différentes spécialités. En déplaçant la gestion du risque au niveau des industries de l'aval au détriment des élevages, l'intégration verticale a favorisé la concentration spatiale des productions animales. Le cas des Etats-Unis illustre bien ce phénomène.

Aux Etats-Unis, à partir des années 1980 , le secteur de la volaille est devenu de plus en plus intégré verticalement et la part de la volaille produite sous forme de contrat a augmenté. Cette évolution industrielle s'est traduite par une concentration géographique dans le SudEst des Etats-Unis de l'appareil productif et de la transformation (Goodwin 2005). Cette agglomération de la production de volaille a eu lieu dans des régions bénéficiant d'un bon accès à la production de céréales et de soja. Selon l'auteur, plus de la moitié de la production de poulets de chair des Etats-Unis se concentre sur 77 comtés, soit 2,5\% des comtés et $90 \%$ de la production sur $10 \%$ des comtés. En 2004, 47\% de la production de l'industrie de la volaille était assurée par 3 entreprises. Cette part a doublé en vingt ans. La taille moyenne des établissements des 8 plus grandes entreprises a plus que doublé entre 1982 et 2002.

En parallèle, comme le rappellent MacDonald et McBride (2009), la plupart des porcs mis sur le marché en 1992 aux Etats-Unis provenaient d'indépendants qui combinaient les différentes étapes de la production (de naisseur à engraisseur). Aujourd'hui, l'activité porcine est concentrée géographiquement et la plupart de la production est organisée par les intégrateurs qui coordonnent la production entre les producteurs spécialisés en plusieurs étapes distinctes. Certains intégrateurs organisent la production de porcs exactement comme les intégrateurs de poulets de chair. Environ 40 grands intégrateurs coordonnent désormais $75 \%$ des 100 millions de porcs commercialisés chaque année aux Etats-Unis.

A l'inverse, la filière lait des EtatsUnis est moins intégrée verticalement et la production de lait y reste plus dispersée géographiquement que les autres productions animales. L'organisation de la filière laitière a peu évolué car la plupart des fermes vendent encore leur lait à travers des coopératives laitières, ce qui peut expliquer pourquoi cette production demeure la moins concentrée géographiquement (Herath et al 2005). En effet, $70 \%$ des producteurs laitiers, ce qui représente $76 \%$ de la production 
de lait, expédient leur lait aux usines liées à leurs propres coopératives. Notons que la production laitière est également moins concentrée spatialement que la production de viande. En effet, certaines exploitations bovines sont détenues par l'industrie avale ou des entreprises spécialisées dans l'élevage bovin possédant leur propre usine d'aliments pour bétail.

Le secteur de la volaille en France est également un bon exemple. En effet, la filière française de volailles de chair est fortement intégrée et est fortement concentrée spatialement. Elle est organisée autour d'un nombre limité de groupes transformateurs et de nombreux éleveurs avec des contrats d'intégration. Plus de $90 \%$ des éleveurs ont des contrats d'intégration si on inclut les contrats coopératifs, $75 \%$ si on les exclut. Plus de $60 \%$ de la production de volaille est concentrée dans les régions Bretagne et Pays de la Loire.

\section{2 / Régulation environne- mentale et relocalisation des productions animales}

La concentration géographique de la production animale, en se traduisant par une concentration géographique des effluents issus de ces élevages, peut devenir une source importante de pollutions (voir sur la figure 1 la répartition spatiale des rejets d'azote organique en 2010). En 2000, les exploitations spécialisées en production porcine détenant de 40 à 60 porcs/ha épandaient de 720 à $910 \mathrm{~kg}$ d'azote par hectare (Ilari et al 2003). Dans les années 1990, selon les données Agreste, la forte concentration s'est opérée à un rythme soutenu dans les exploitations porcines tandis que les structures foncières sont restées assez stables pendant cette période. Aux EtatsUnis, les exploitations de plus de 1000 places, qui représentent $2 \%$ des élevages, détiennent $35 \%$ du potentiel de production mais seulement $2 \%$ des surfaces agricoles (Tregaro et Lossouarn 2004).

L'Union Européenne a, au travers de la directive «Nitrates», cherché à limiter la pollution des eaux liée à une trop forte concentration en nitrates d'origine agricole. Son application en France s'est traduite par la mise en place d'un niveau maximum d'azote par hectare et par an $(170 \mathrm{~kg} / \mathrm{ha} / \mathrm{an})$. Cette restriction des droits d'épandage a logiquement engendré des difficultés dans les régions à forte densité animale et où les surfaces disponibles ou accessibles pour l'épandage n'étaient pas suffisantes en raison des coûts non négligeables notamment de transport ou de traitement que cela a induit.

Dans ce qui suit, nous évaluons, tout d'abord, si les réglementations environnementales ${ }^{5}$ ont permis d'éliminer les excès d'azote dans certains territoires, comme la Bretagne. Ensuite, nous nous interrogeons si les mesures prises en matière de régulation environnementale (notamment, les contraintes sur les surfaces d'épandage qui réintroduisent un lien au foncier) peuvent conduire à la dispersion géographique de la production. Autrement dit, si la politique environnementale, telle qu'elle est appliquée peut contrebalancer les forces des marchés poussant à l'agglomération des productions. Enfin, nous discuterons des effets possibles des changements structurels dans le secteur agricole sur la situation environnementale des territoires avec forte densité animale.

\section{1 / Décalage persistant entre les besoins en azote et l'azote organique issu des productions animales}

La spécialisation des exploitations et des territoires dans les productions animales a en contrepartie fait émerger des territoires présentant des excédents d'azote comme en Bretagne. Par exemple, en 2008, les besoins totaux en azote de la Bretagne sont de l'ordre de 214 milliers de tonnes tandis que les effluents en apportent environ 169 milliers de tonnes et 100 milles tonnes d'azote minéral sont achetées, d'où un excédent de 57 milliers de tonnes (tableaux 4 et 5). Le tableau 4 montre que si la situation environnementale globale s'est amélio-

Tableau 4. Bilan en azote de la Bretagne (méthode «bilan Corpen»).

\begin{tabular}{|c|c|c|}
\hline & 2001 & 2008 \\
\hline $\begin{array}{l}\text { Surface Agricole Utile (ha SAU) } \\
\text { Surface Potentiellement Epandable (ha SPE) }\end{array}$ & $\begin{array}{l}1701566 \\
1191096\end{array}$ & $\begin{array}{l}1661332 \\
1162932\end{array}$ \\
\hline $\begin{array}{l}\text { Nombre de cantons en ZES* } \\
\text { Part des exploitations en ZES }\end{array}$ & $\begin{array}{c}104 \\
64\end{array}$ & 90 \\
\hline $\begin{array}{l}\text { [1] Azote organique (t) } \\
\text { dont Bovin } \\
\text { dont Porcin } \\
\text { dont Avicole } \\
\text { [2] Azote organique résorbé } \\
\text { [3] Azote organique net ([1] - [2]) }\end{array}$ & $\begin{array}{c}226900 \\
121622 \\
61354 \\
39954 \\
11500 \\
215400\end{array}$ & $\begin{array}{c}204288 \\
110831 \\
64356 \\
29101 \\
35578 \\
168710\end{array}$ \\
\hline $\begin{array}{l}\text { Azote organique [1]/SPE }(\mathrm{kg} / \mathrm{ha}) \\
\text { Azote organique [1] /SPE }(\mathrm{kg} / \mathrm{ha}) \text { dans ZES } \\
\text { Azote organique net [3] /SPE }(\mathrm{kg} / \mathrm{ha})\end{array}$ & $\begin{array}{l}190 \\
216 \\
181\end{array}$ & $\begin{array}{l}176 \\
145\end{array}$ \\
\hline [4] Apport en azote minéral (t) & 120600 & 100035 \\
\hline $\begin{array}{l}\text { [5] Besoin en azote }(\mathrm{t}) \\
\text { Besoin en azote organique [5] - [4] } \\
\text { [6] Excédent d'azote }([3]+[4]-[5]) \\
\text { Excédent d'azote en } \%[6] /[5])\end{array}$ & $\begin{array}{c}237900 \\
117300 \\
103300 \\
43 \%\end{array}$ & $\begin{array}{c}213638 \\
113603 \\
57107 \\
26 \%\end{array}$ \\
\hline
\end{tabular}

*ZES : Zone en Excédent Structurel.

Source : Agreste (ne tient pas compte des effets précédents et des reliquats).

\footnotetext{
5 Voir Peyraud et al (2012) pour une liste détaillée des mesures environnementales pour la maîtrise des pollutions dues à l'élevage.
} 
Tableau 5. Bilan en azote $(N)$ de la Bretagne par département.

\begin{tabular}{|l|c|c|c|c|c|c|c|c|}
\hline & \multicolumn{2}{|c|}{ Côtes d'Armor } & \multicolumn{2}{c|}{ Finistère } & \multicolumn{2}{c|}{ Ille-et-Vilaine } & \multicolumn{2}{c|}{ Morbihan } \\
\hline & 2001 & 2008 & 2001 & 2008 & 2001 & 2008 & 2001 & 2008 \\
\hline SAU (ha) & 453464 & 439366 & 397508 & 393237 & 465568 & 450949 & 385026 & 377780 \\
SPE$^{*}$ (ha) & 317425 & 307556 & 278256 & 275266 & 325898 & 315664 & 269518 & 264446 \\
\hline Nb de cantons en ZES** & 40 & 35 & 29 & 30 & 19 & 13 & 16 & 12 \\
Part des expl. en ZES & $83 \%$ & & $70 \%$ & & $46 \%$ & & $58 \%$ & \\
\hline N org. (t) & 66907 & 62166 & 58358 & 53077 & 53411 & 46920 & 48236 & 42125 \\
N org. Résorbé (t) & & 15130 & & 11885 & & 1681 & & 4882 \\
N org /SPE (kg/ha) & 211 & 202 & 210 & 193 & 164 & 149 & 179 & 159 \\
N org /SPE en ZES (kg/ha) & 222 & & 235 & & 191 & & 206 & \\
N org net/SPE & & 153 & & 150 & & 143 & & 141 \\
\hline Apport N minéral & & 23990 & & 28271 & & 27192 & & 20582 \\
Besoin N (t) & & 56537 & & 47880 & & 64877 & & 44344 \\
\hline Excédent d'azote & & 14489 & & 21583 & & 7554 & & 13481 \\
Excédent d'azote (\%) & & $25,6 \%$ & & $45,1 \%$ & & $11,6 \%$ & & $30,4 \%$ \\
\hline
\end{tabular}

*Surface Potentiellement Epandable ; **Zone en Excédent Structurel.

Source Agreste (ne tient pas compte des effets précédents et des reliquats).

rée, la Bretagne a toujours de nombreux cantons en excédent d'azote en 2008. La baisse des excédents d'azote est très concentrée au sein du territoire breton car seuls 14 cantons sur 104 ne sont plus classés en zone d'excédent structurel (ZES) entre 2000 et 2008. Malgré les efforts effectués en Côtes d'Armor et dans le Finistère, liés surtout à la multiplication des stations de traitement des effluents (IFIP 2006), les excédents d'azote sont toujours principalement localisés dans les cantons de ces deux départements. Par ailleurs, si on observe une baisse de l'excédent d'azote en Bretagne entre 2001 et 2008, celle-ci est largement imputable à la baisse d'environ 20000 tonnes d'azote organique issu des productions animales car, en même temps, le besoin en azote organique n'a que très légèrement diminué. La baisse de l'excédent d'azote est surtout liée à la diminution des effectifs dans le secteur avicole et, dans une moindre mesure, dans le secteur bovin mais aussi à une hausse des prix des fertilisants chimiques (figure 2). La quantité d'azote organique produite par le secteur porcin a augmenté en dépit de la généralisation de l'alimentation biphase (permettant d'ajuster les apports nutritionnels aux besoins réels de l'animal lors de différents stades de son développement) dans les élevages ${ }^{6}$. Par ailleurs, on aboutit à une situation paradoxale en Bretagne, dans la mesure où on élimine de l'azote dans l'ouest de la Région alors que dans l'est de la Bretagne la production d'azote est inférieure au besoin.

Il est donc très clair que l'application de la réglementation environnementale n'est pas suffisante pour réduire les excès d'azote et la concentration spatiale des productions animales. Au moins deux raisons potentielles peuvent être avancées pour expliquer la persistance d'excès d'azote dans de nombreux cantons en France, vingt ans après l'apparition de la directive «Nitrates». Tout d'abord, la réglementation n'est pas respectée. Ensuite, les coûts associés à la réglementation ne sont pas dissuasifs pour changer les pratiques des éleveurs. Les deux explications sont vraisemblablement valables'. Les gains à l'agglomération sont tels que les coûts associés aux respects des contraintes environnementales sont insuffisants pour affecter substantiellement une relocalisation des productions agricoles.

\section{2 / La localisation des produc- tions animales est-elle sensible aux différentiels de coûts dus à la réglementation?}

De nouveau, l'expérience du Canada et des Etats-Unis est intéressante. En effet, il y existe une mesure du degré de sévérité de l'application de la réglementation environnementale par les différentes collectivités locales (les exigences environnementales, les contraintes sur les plans d'installation et de fertilisation, les niveaux de contrôle, etc. varient selon les territoires). Autrement dit, le coût du respect des contraintes environnementales varie selon les territoires. Cette mesure a été utilisée pour analyser l'impact du degré de sévérité, et donc des coûts associés à la réglementation environnementale, sur la distribution spatiale des productions animales.

\footnotetext{
6 En 2008, $81 \%$ des places de truies et $83 \%$ des places d'engraissement sont en alimentation biphase contre respectivement 65 et $59 \%$ en 2001 selon 1 'enquête Bâtiment Elevage (Agreste).

7 Rappelons que des aides publiques (PMPOA 1 et 2) ont été accordées pour la mise aux normes à des exploitations qui ne respectaient pas la réglementation environnementale. Ceci peut illustrer le caractère peu incitatif de l'action publique dans le cadre de la maîtrise des pollutions des eaux.
} 
Figure 2. Indice des prix «Engrais et amendements» (Base 100 en 2005). Source : Institut national de la statistique et des études économiques (Insee) - Département synthèses sectorielles.

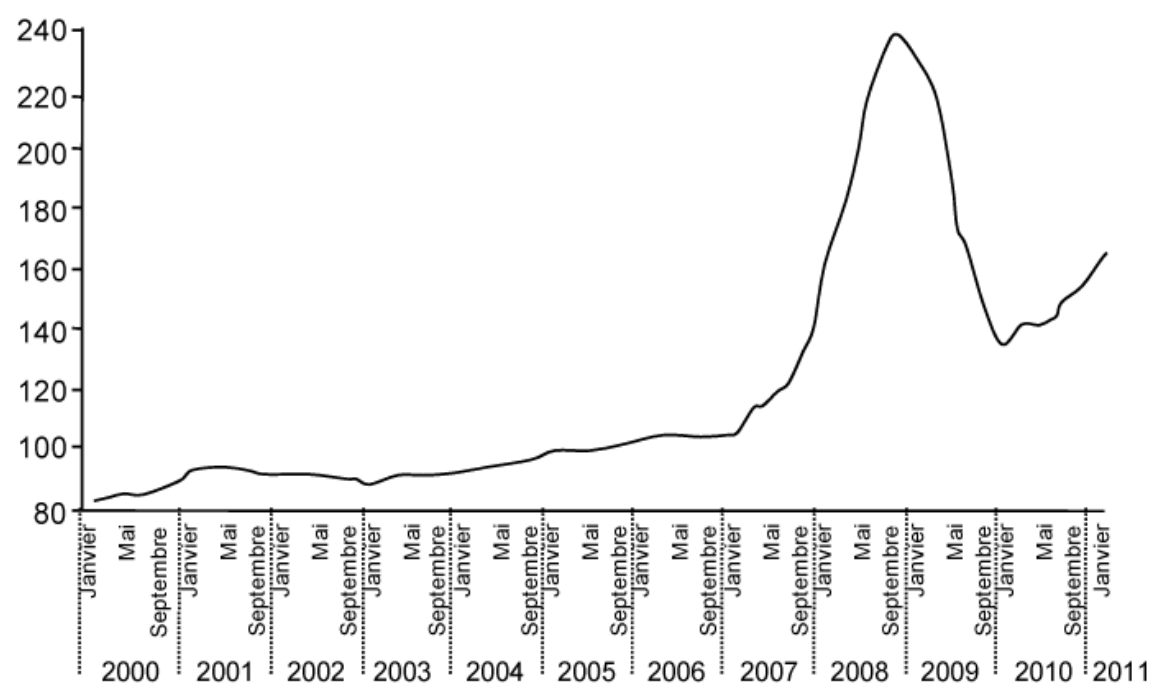

A partir de données canadiennes, Weersink et Eveland (2006) montrent que les différences entre les communes en matière de sévérité pour le respect des règlements environnementaux n'influencent pas le nombre d'installations et le nombre d'autorisations d'expansion des élevages. Ce dernier augmente là où le nombre d'éleveurs est élevé, suggérant le rôle important des économies d'agglomération. Par ailleurs, Metcalfe (2001), à partir de données permettant d'identifier le degré de sévérité de l'application de la réglementation environnementale, montre que la localisation des élevages porcins de grande taille n'est pas affectée par les différences spatiales dans les coûts liés à la politique environnementale. En revanche, ces différences de coûts ont affecté la distribution spatiale de la production porcine des exploitations de taille modeste. Plus précisément, l'accroissement de la sévérité aux EtatsUnis à la fin des années 1990 (Metcalfe 2001) a surtout affecté négativement les petites exploitations. En revanche, en contrôlant mieux le rôle des économies d'agglomération, les études de Roe et al (2002) et Isik (2004) suggèrent que les effets de la réglementation environnementale sont globalement négatifs pour les productions porcines et laitières. Par ailleurs, Herath et al (2005) confirment que le niveau de sévérité de l'application de la politique environnementale, selon les Etats, influence la localisation des productions animales, plus particulièrement la production porcine et laitière. Néanmoins, ces études montrent que les économies d'agglomération liées à la proximité des industriels structurent davantage l'organisation spatiale des productions animales. Il apparaît donc que l'action publique n'est pas en mesure de contrecarrer les concentrations spatiales excessives des productions animales.

\section{3 / La disponibilité foncière limite-t-elle la concentration spa- tiale des productions animales?}

En fixant une valeur maximale à la quantité d'azote organique pouvant être épandue par hectare de surface épandable, la concurrence pour la terre permettant d'épandre les excès d'effluents devrait favoriser la dispersion géographique des productions animales. En effet, les exploitations ayant une production animale, et donc d'effluent, supérieure à leur surface en terre doivent trouver des terres supplémentaires en dehors de leur exploitation pour épandre le lisier. Ainsi, les éleveurs ayant des excédents d'effluents doivent louer la possibilité d'épandre aux agriculteurs ayant des capacités d'accueil et doivent assurer le transport et l'épanvironnementale génère des coûts de location de terre ainsi que des coûts de transport et d'épandage. Les études empiriques montrent que ces coûts ne sont pas négligeables. Même si les prix des transactions sur les services d'épandage ne peuvent être observés, on peut les révéler indirectement en s'intéresdage sur le prix des terres agricoles. A partir d'une analyse hédoniste sur une base de 7000 transactions foncières en Bretagne, Le Goffe et Salanié (2005) montrent que le prix de la terre agricole croît, toutes choses égales par ailleurs, avec la densité animale ou avec la densité de porcs. Par ailleurs, les coûts monétaire et en temps liés à l'épandage (chargement, transport et application) dage. Autrement dit cette mesure ensant à la capitalisation de la rente d'épan- ne sont pas négligeables. Les coûts de transport sont estimés de 1,50 à $4,50 €$ par $\mathrm{kg}$ d'azote pour le porc et de 0,40 à $1 €$ pour la volaille en fonction de la distance parcourue (Djaout et al 2009). Smith et al (2006) ont évalué ce coût à 2,13 \$CAN par tonne de lisier pour les exploitations porcines et laitières et à 4,28 \$CAN/t pour les exploitations avicoles. En conséquence, la contrainte d'épandage introduite par la directive «Nitrates» dans les zones à excédent d'azote implique des coûts croissants avec la densité animale, car les éleveurs devront payer un droit d'épandage plus élevé ou épandre de plus en plus loin au fur et à mesure que la production locale s'accroît. Cette contrainte devrait donc décourager l'agglomération de la production animale et, au contraire, induire son éparpillement spatial.

L'étude de Kaplan et al (2004) analysant les effets de la contrainte d'épandage liée à la terre sur la localisation des productions agricoles est intéressante. Elle concerne 34 Etats des Etats-Unis d'Amérique qui ont décidé de limiter les taux d'application du lisier sur les surfaces agricoles. Plus précisément, la contrainte vise à ce que la production de fertilisant organique issue des animaux n'excède pas les besoins en fertilisant des cultures de la région. Les auteurs simulent l'impact, sur les niveaux de production animale et végétale par Etat, de la mise en place d'une contrainte concernant l'application du lisier sur les terres agricoles, en considérant différents taux de substitution entre les fertilisants chimiques et organiques (de 20 à 40\%). Si l'on considère un taux de substitution de $20 \%$ (taux moyen aux USA) et un coût lié à la gestion du lisier, l'ensemble des productions animales baissent, surtout dans le secteur de la volaille (- 7\%) et dans une moindre mesure dans les secteurs de la viande bovine et porcine (respectivement - 2,20 et - 1,56\%). La production de maïs baisse également tandis que la production de blé augmente. En effet, la production de volaille requiert des quantités relativement importantes de maïs et cette culture est produite dans des régions peu spécialisées dans la volaille. En revanche, le blé étant relativement plus consommateur de phosphore, la mise en œuvre de la contrainte accroît la demande de blé pour l'épandage. Par ailleurs, cette baisse de la production animale s'accompagne d'une relocalisation des productions des régions spécialisées et intensives vers les Etats de la «Corn Belt». Autrement dit, les contraintes d'application du lisier sur les surfaces agricoles induisent une dispersion des productions animales vers les régions spécialisées dans les productions végétales. 
Or, l'étude des données françaises montre que la disponibilité foncière n'est pas un frein à l'agglomération (Ben Arfa et al 2010, Gaigné et al 2011). Pour tenter de comprendre la non-influence de l'application locale de la norme environnementale, il faut prendre en compte la possibilité que peuvent avoir les éleveurs (et notamment les plus performants) de faire évoluer leur technologie pour atténuer les effets négatifs de la contrainte environnementale sur leur productivité (par réduction de leurs effluents ou par modification de la nature de leurs gestions des effluents). Cette réaction est conforme à l'hypothèse dite «de Porter» développée par Porter et van der Linde (1995). Bien qu'assez controversée (cf. Ambec et Barla 2005), cette hypothèse stipule que, sous certaines conditions et en dépit des surcoûts qu'elles induisent, les contraintes environnementales peuvent inciter à l'innovation et entraîner des changements de "trends» technologiques bénéfiques en termes de compétitivité pour les entreprises qui s'y engagent. Cette vision «win-win» des relations entre les actions environnementales et les intérêts économiques semble se traduire, dans notre cas, par la poursuite des mouvements antérieurs de concentration géographique des productions animales en France.

Par ailleurs, en France, les exploitations sont autorisées à éliminer les excès d'azote en procédant à son traitement ${ }^{8}$. Ainsi, la région Bretagne concentre 81\% des volumes de lisiers traités alors que cette région représente $52 \%$ des exploitations et $60 \%$ des places selon l'Enquête cheptel porcin de novembre (Agreste 2008 cité dans Robreau et al 2010). Rappelons que plus de $80 \%$ des exploitations porcines en Bretagne sont situées en ZES. Or, les technologies de traitement sont caractérisées par des charges fixes élevées. Selon une étude de l'IFIP (IFIP 2006), l'introduction d'une station de traitement implique un surcoût d'environ $10 €$ par porc, soit 0,08 à $0,10 € / \mathrm{kg}$ de porc $^{9}$. Cette analyse technico-économique repose sur l'étude du poste «déjections») de 39 élevages de porcs. Les volumes traités par an sont en moyenne de $8000 \mathrm{~m}^{3}$. Le coût de fonctionnement (électricité, additifs, main-d'œuvre...) est en moyenne de 2,60€ par $\mathrm{m}^{3}$ traité tandis que l'investissement moyen est $46 €$ par $\mathrm{m}^{3}$ (de 25 à $\left.86 €\right)$. L'investissement de départ est donc important. Ainsi, le coût moyen de traitement décroît avec la quantité de lisier traitée. Autrement dit, le traitement peut être mis en place par les élevages de grande taille et peut accroître la taille des élevages. Même si la mutualisation des stations de traitement est possible, le traitement est le plus souvent réalisé au niveau de l'exploitation $(75 \%$ du lisier traité et $68 \%$ des exploitations selon l'Enquête cheptel porcin de novembre 2008).

Le travail de Smith et al (2006) démontre bien l'intérêt de considérer le traitement des effluents comme une voie alternative à la gestion des effluents. En effet, leurs résultats contrastent avec les études antérieures qui considéraient seulement l'épandage sur le sol pour résorber les effluents. La prise en compte de la possibilité de traiter les effluents apporte des nouveaux éclairages comme l'illustrent deux travaux conduits sur des données françaises. Djaout et al (2009) en s'intéressant à l'adaptation des élevages aux contraintes de la directive «Nitrates» en Côtes d'Armor, montrent que les élevages les plus efficaces sont incités à traiter leurs effluents tandis que les élevages les moins efficaces sont incités à réduire leur cheptel. Par ailleurs, Gaigné et al (2011) montrent que le recours au traitement du lisier peut accroître la concentration spatiale de la production porcine en favorisant une réallocation de la production des petites exploitations vers les plus grandes.

L'application de la directive «Nitrates» en France ne réduit pas la concentration spatiale excessive des productions animales en raison des technologies de traitement du lisier favorisant la concentration spatiale des productions animales. Les subventions publiques en France pour les élevages localisés dans les ZES mettant en place des stations de traitement a donc favorisé principalement les grandes exploitations (au détriment des plus petites) qui sont à la fois à l'origine des excédents d'azote et en mesure de supporter les charges fixes liées au traitement.

\section{3 / Quel avenir ?}

Nous avons vu que l'application de la directive «Nitrates» en France avait eu peu d'effets sur les excès d'azote dans les territoires de forte densité animale. Les incitations créées par la puissance publique à travers sa politique environnementale ne sont pas suffisamment puissantes pour améliorer significativement la situation environnementale des bassins de production animale en excédent d'azote. Les mécanismes de marché et le premier pilier de la PAC sont beaucoup plus structurants dans l'organisation économique et spatiale des filières animales. Or, le secteur agricole en Europe entre dans une nouvelle ère qui ne sera pas sans conséquences sur les niveaux de pollution selon les territoires. Deux événements méritent une attention particulière : i) la montée du prix de l'énergie et ii) la dérèglementation des marchés agricoles.

\section{1 / Le prix de l'énergie : vers une meilleure valorisation des effluents d'élevage?}

La baisse du prix relatif de l'énergie (notamment par rapport au prix du travail), pour les raisons évoquées plus hauts, joue un rôle important dans la persistance des problèmes environnementaux. La principale source d'azote des cultures est donc fournie par les engrais minéraux. Une grande partie des effluents d'élevage ne trouve donc pas d'usage fertilisant. Or, on a vu en début de cette section que la baisse en 2008 de la consommation d'engrais minéral est imputable à la hausse de son prix (figure 2). Dans un contexte où le prix de l'énergie va vraisemblablement augmenter, l'accroissement possible des coûts de transport et des prix des fertilisants chimiques peut constituer un puissant levier favorisant une meilleure valorisation des matières organiques.

Ce qui compte dans les choix des producteurs, ce sont les prix relatifs entre les différents fertilisants et la quantité de travail nécessaire par type de fertilisant pour son application. Autrement dit, l'utilisation croissante de l'engrais organique requiert non seulement des prix relativement élevés des engrais minéraux mais aussi une gestion des engrais organiques relativement économe en travail. Pour ce dernier point, les évolutions technologiques sont cruciales. Or, on voit déjà émerger de nouveaux types de producteurs d'engrais à partir des effluents d'élevage et déchets des industries agroalimentaires. Les industriels proposent des nouvelles technologies avec une phase de méthanisation des déchets organiques et une phase d'extraction de l'azote, du phosphore et de la potasse des déchets pour les transformer en engrais et les expédier vers les régions de cultures. D'autres solutions

\footnotetext{
${ }^{8}$ Les procédés de traitement sont nombreux. La réduction ou la suppression de la fraction azotée contenue dans les lisiers peut s'effectuer par différents procédés (traitement biologique par transformation des nitrates en azote gazeux, concentration de l'azote dans des produits minéraux facilement exportables, compostage...).

${ }^{9}$ Le Centre d'Economie Rurale des Côtes d'Armor aboutit à des résultats proches. Le traitement du lisier coûte entre 6,31 $€$ par $\mathrm{m}^{3}$ pour des stations à traitement simplifié et $10,49 €$ dans le cas d'un traitement complet.
} 
techniques ont été récemment mises en œuvre. Il s'agit de bâtiments d'un nouveau genre permettant de récupérer plus facilement les effluents pour pouvoir ensuite fabriquer des engrais organiques. Les solutions techniques et économiquement rentables semblent permettre une plus grande utilisation des engrais organiques. Dans tous les cas, ces solutions, permettant une meilleure valorisation des effluents et une réduction des excédants de nitrates dans les territoires d'élevage, ne favorisent pas la dispersion des productions animales. En effet, l'intérêt de ces solutions est d'exporter le lisier sous forme d'engrais organique pour ne pas être limité par la quantité de terre disponible pour l'épandage. Au contraire, ces innovations technologiques risquent de renforcer le processus d'agglomération des productions animales. La production d'engrais organique impliquant des charges fixes, cette activité n'est rentable que si l'on produit à grande échelle.

Par ailleurs, dans une perspective de prix élevés de l'énergie, le bilan énergétique des différents systèmes de production sera décisif (Dupraz et al 2010). Les techniques permettant d'économiser de l'énergie sans accroître significativement la quantité de travail seront favorisées. Par rapport à notre question, il est intéressant de distinguer les bilans énergétiques des systèmes intensifs et extensifs. Par exemple, les élevages laitiers intensifs, qui utilisent beaucoup plus d'énergie que les systèmes plus extensifs optimisant le pâturage, seront défavorisés (Dupraz et al 2010). Toutefois, dans le cas du lait, cela ne va pas forcément induire la dispersion géographique de la production. Pour cela, il faut que les gains en énergie deviennent supérieurs aux économies de taille au niveau des exploitations et des territoires.

\section{2 / Dérèglementation des mar- chés agricoles : vers une plus gran- de concentration de la production ou une plus grande autonomie alimentaire des territoires ?}

Ensuite, la poursuite de la dérégulation des marchés agricoles aura des répercussions non négligeables sur l'organisation économique et spatiale dans les secteurs subissant la déréglementation, mais aussi dans les autres secteurs des productions animales en raison de leurs interdépendances, notamment à travers le marché foncier. Rappelons que la baisse du prix relatif de l'énergie (et donc du transport et des intrants chimiques) a favorisé la concentration géographique des élevages et la dissociation régionale des productions végétales et animales pour les raisons évoquées plus haut. La principale source d'azote des cultures est donc fournie par les engrais minéraux tandis que les protéines nécessaires à l'alimentation animale sont issues dans une large mesure des importations de protéagineux. Or, s'il est aisé de prévoir que la déréglementation des marchés agricoles va affecter l'organisation spatiale des filières animales, il est plus difficile de prévoir dans quel sens cela va modifier la structuration des territoires : vers une accentuation de l'agglomération des productions animales et la spécialisation des territoires ou vers un retour de bassins de production avec élevage et production végétale.

Toutes choses égales par ailleurs, la concentration spatiale des productions animales se renforcera avec la fin du soutien de la PAC à certaines catégories d'éleveurs. Nous avons vu plus haut que les secteurs fortement soutenus par la PAC sont moins concentrés géographiquement. Or, la suppression du régime des quotas laitiers prévue à l'horizon 2015 modifiera sensiblement la localisation de la production de lait en France (Perrot et Chatellier 2009, Chatellier et Dupraz 2011). Cette politique, par ses mesures, avait figé dans l'espace l'offre territoriale de lait. C'est une des raisons pour laquelle le secteur du lait apparaît moins concentré que les secteurs dit «hors-sol» (tableau 3). Or, à partir de 2015 , les règles administratives décidées par les pouvoirs publics ne dicteront plus les ajustements de l'offre de lait à la demande. On peut donc s'interroger sur les effets à venir de la fin programmée du soutien du secteur du lait sur la réorganisation spatiale et économique de cette filière. La fin des quotas ainsi qu'un éventuel découplage de la PMTVA peuvent provoquer davantage d'agglomération. Le niveau de décision sera décentralisé et, de fait, reviendra aux industriels de la transformation. Des systèmes de contractualisation entre éleveurs et industriels vont se développer (Lelyon et al 2012). Au vu des mécanismes évoqués plus haut, l'inégale répartition de la production de lait ainsi que des transformateurs devrait s'accentuer au profit des bassins les plus compétitifs et au détriment des petites exploitations. La baisse du nombre d'exploitations dans l'élevage et la polycultureélevage, surtout celle des petits élevages producteurs de lait, et la progression des grands élevages observées dans le dernier Recensement Agricole 2010 devraient se poursuivre. Ce mouvement de polarisation aura également des conséquences sur les autres secteurs partageant les mêmes ressources (l'alimentation animale et la terre pour l'épandage). Sur ce dernier point nous manquons cruellement de travaux de recherche. Dans tous les cas, le renforcement de l'intégration verticale dans la production du lait et le poids croissant des forces de marché dans la régulation de ce secteur vont vraisemblablement intensifier la volonté de regrouper géographiquement les différents acteurs de la fillière laitière et d'accroitre la taille de ses producteurs et transformateurs. Cette tendance rend plus que nécessaire de mener une politique environnementale stricte et efficace.

Toutefois, la dérèglementation des marchés agricoles associée à une hausse des prix de l'énergie et de la demande alimentaire mondiale ont fortement modifié les paramètres influençant les choix des éleveurs. En effet, ces changements ont engendré un accroissement du niveau et de la volatilité des prix des céréales ainsi qu'une accentuation des risques de défauts d'approvisionnement. Ces changements structurels concernant le marché des céréales peut constituer un puissant levier favorisant le retour de territoires mixtes en termes de production animale et végétale. En effet, si le recul de l'élevage dans les régions dominées par les productions céréalières persistera vraisemblablement, les zones d'élevage peuvent connaître un développement des activités céréalières. En effet, dans un souci de sécuriser leur approvisionnement pour nourrir leurs animaux (en termes de coût, quantité et qualité), les éleveurs sont davantage incités à développer des exploitations mixtes. La production de céréales au sein des élevages peut être un moyen de maîtriser les coûts liés à l'alimentation animale et de s'assurer de disposer des quantités suffisantes de matières premières pour nourrir les animaux dans un contexte d'incertitude croissante sur les prix et les quantités disponibles de céréales. Cependant, les gains associés à l'autonomie des systèmes alimentaires dans un contexte de fortes tensions sur les marchés des céréales devront être élevés pour compenser les gains à la spécialisation.

\section{3 / Faut-il réguler les industriels des filières animales?}

La faible efficacité des politiques environnementales incite à s'interroger sur la manière dont les politiques devraient être menées ${ }^{10}$. Le secteur de l'élevage, par ses caractéristiques, rend difficile la réduction des excès d'azote par le biais des politiques environnementales. Tout

\footnotetext{
10 Rappelons que plus de 560 millions d'euros d'aides publiques ont été versés à plus de 45000 éleveurs dans les zones vulnérables dans le cadre des mesures PMPOA.
} 
d'abord, la pollution d'origine agricole des eaux est une pollution diffuse. Le régulateur ne peut pas observer précisément la contribution individuelle des éleveurs dans les excès d'azote. Il est par exemple très difficile de mesurer directement les émissions individuelles dans les nappes phréatiques. Ensuite, les producteurs sont très nombreux, près de 500000 exploitations professionnelles en France selon le recensement agricole de 2010 (dont près de la moitié concernant l'élevage). Ce très grand nombre d'éleveurs rend l'administration des politiques environnementales très coûteuse. Et si ce n'est pas le cas, cela se traduit par une probabilité de subir un contrôle qui est relativement faible (ce qui devrait être moins vrai pour les plus grandes exploitations, soumises à la réglementation des exploitations classées). Autrement dit, rien ne garantit que le niveau d'effort souhaité au niveau des éleveurs pour réduire les excès d'azote soit atteint.

En conséquence, il convient de s'interroger sur la ou les cibles des mesures liées à la régulation environnementale : le pollueur ou l'acquéreur du bien produit par le pollueur (dans notre cas la coopérative ou les industries d'abattage, découpe et transformation...). La question est légitime car les industriels s'approvisionnent massivement auprès d'éleveurs localisés dans les cantons classés en ZES. Or, des travaux récents posent la question du maillon le plus pertinent le long de la chaîne verticale sur lequel doit s'exercer la régulation environnementale (Aggarwal et Lichtenberg 2005, Metcalf et Weisbach 2009, Bushnell et Mansur 2011, Mansur 2011). L'objectif est de déterminer s'il est préférable de réguler l'industrie polluante ou les industries se situant en amont ou en aval de ces industries polluantes («vertical targeting»). C'est le fondement même du principe «Pollueur Payeur» qui est ici en discussion. Dans certains cas, il est souhaitable de réguler l'acheteur du bien produit par le pollueur. Ces travaux concluent qu'il peut être plus efficace de cibler plutôt l'acheteur du bien produit par le pollueur que le pollueur luimême. Dans le cas de l'élevage, on peut ainsi se demander s'il est plus efficace de cibler les mesures sur l'éleveur ou sur l'industrie des filières animales (coopératives, industrie d'abattage, de découpe, de transformation, laiterie...).

Ces travaux montrent tout d'abord, que le niveau de la filière où il y a relativement moins d'acteurs doit être privilégié. Comme le nombre d'éleveurs est bien plus grand que le nombre d'industriels, les coûts associés à l'administration de la politique liée à la directive nitrates seraient significativement plus faibles si la régulation portait sur la demande des productions animales et non sur l'offre. Comme on l'a vu plus haut, les dix premiers groupes dans l'industrie de la viande de volaille représentent $80 \%$ du chiffre d'affaire du secteur et dix groupes seulement transforment $75 \%$ du lait produit en France. De même, $80 \%$ de la production porcine en Bretagne sont détenues par cinq organisations de producteurs (qui sont très intégrées verticalement).

Ensuite, il peut être préférable de réguler le segment de la filière où le degré de concurrence est faible. D'une part, les taxes peuvent être inférieures au dommage environnemental marginal pour un même niveau d'efficacité environnementale lorsque l'industrie a un pouvoir de marché (Canton et al 2008). D'autre part, l'économie géographique montre que ce sont essentiellement les secteurs en concurrence imparfaite qui bénéficient des économies d'agglomération (Fujita et Thisse 2002). Nous avons vu plus haut que les industries des filières animales n'échappent pas à ce constat. Autrement dit, une taxe environnementale appliquée aux industriels, des filières animales ne ferait que capter la rente dont bénéficient ces industriels, due à la concentration spatiale des productions animales. En effet, rappelons que si l'agglomération des élevages sur quelques cantons est à l'origine des excès d'azote, cette agglomération permet de réduire les coûts de production des industriels des filières animales.

Par ailleurs, lorsque les éleveurs et les industriels sont liés par des doubles contrats (ces derniers fournissent des intrants aux éleveurs qui vendent leur production à ces industriels), Aggarwal et Lichtenberg (2005) montrent que la taxe optimale sur la pollution doit porter sur les éleveurs et les industriels. Dans une situation d'imperfections de marché, il existe des interactions entre les décisions des producteurs et de leurs clients qui ne sont pas internalisées. Dans ces circonstances, si une taxe environnementale est seulement appliquée sur l'activité d'élevage, alors cette taxe est sous optimale. En effet, en fixant les termes du contrat avec les éleveurs, les transformateurs n'internalisent pas l'impact de leur choix sur la situation environnementale liée à l'activité d'élevage. Une taxe environnementale appliquée également aux industriels de la transformation est souhaitable pour garantir une diminution des excès de pollution.

$\mathrm{Au}$ vu des spécificités des industries des filières animales, la régulation environnementale concernant les pollutions liées aux excès d'azote pourrait être plus efficace si elle ciblait également les industries avales. On pourrait par exemple prélever une taxe sur les industriels des filières animales en fonction du nombre d'animaux ou de la quantité de lait en provenance des cantons classés en ZES. L'information est déjà disponible pour les industries de la viande car les mouvements des animaux sont répertoriés. La provenance et la destination des animaux sont connues. De plus, cela permettrait d'inciter davantage les industriels des filières animales, qui, rappelons-le, bénéficient également des économies d'agglomération, à mettre en place des technologies valorisant mieux les rejets d'azote et de phosphore.

Toutefois, le choix du niveau d'intervention dans la filière doit minimiser les «fuites», c'est-à-dire ne pas favoriser l'importation en provenance d'autres bassins de productions dotés de modes de production moins polluants ou une relocalisation des industries dans des pays avec une politique environnementale plus laxiste. Or, il est montré que, lorsque les marchés internationaux sont intégrés, ces fuites sont réduites si les industries d'aval ou d'amont sont taxées au lieu de l'activité qui est à l'origine de la pollution (Bushnell et Mansur 2011). Sur ce dernier point, les travaux scientifiques sont peu développés et méritent d'être approfondis afin d'évaluer la robustesse de ces premiers résultats. Evidemment, un des enjeux est de savoir si une politique environnementale visant uniquement les acteurs (agriculteurs, transformateurs, consommateurs) implantés sur le territoire est par elle-même efficace ou si des mesures complémentaires comme des taxes à la frontière ou des normes environnementales sont nécessaires.

\section{Conclusion}

Dans cet article, nous avons mis en avant les mécanismes provoquant l'agglomération des productions animales et les excès d'azote au niveau des territoires. Les facteurs physiques comme le climat et la disponibilité des terres ont donc un rôle moins important dans la localisation des productions agricoles en raison des changements technologiques dans la production, le transport et la transformation. A l'inverse, les mécanismes de marché affectent de manière croissante l'allocation spatiale des activités des filières animales et impliquent une densification des productions animales, en raison notamment d'une déréglementation en cours des marchés agricoles. Comme souvent, le marché n'internalise pas les effets négatifs environnementaux. D'où les excès d'azote dans de nombreux territoires de l'ouest de la France, grand bassin européen de production animale.

L'accent a été mis sur le rôle du prix de l'énergie ainsi que sur les économies 
d'échelle et les économies d'agglomération dont bénéficient les élevages et les industries des filières animales. Il existe une large littérature dans ce domaine. Même si la présence d'économies d'échelle pour des raisons technologiques au niveau des élevages est parfois remise en cause, d'autres facteurs poussent à l'émergence d'exploitations de grande taille, comme la possibilité de mieux négocier les prix des biens intermédiaires ainsi que la présence d'économies d'échelle au niveau des industries et d'économies d'agglomération au niveau des territoires. Notons également que les restructurations dans les industries se situant à l'aval et à l'amont des élevages sont également responsables de cette concentration spatiale des productions animales.

Notre attention s'est ensuite portée sur la capacité de l'action publique destinée à réduire les excès d'azote, à contrecarrer ces mécanismes poussant à la concen- tration spatiale des rejets d'azote. En l'état actuel de l'action publique, celle-ci n'est pas en mesure de déconcentrer géographiquement les productions animales. $\mathrm{Au}$ contraire, les mesures favorisant le développement du traitement du lisier, la méthanisation ou la production d'engrais organique renforcent le processus de concentration spatiale des activités d'élevage. L'amélioration de la situation du bilan azote dans les années 2000 de la Bretagne est surtout due à une baisse des productions animales, essentiellement dans le secteur avicole, et à une hausse des prix relatifs des engrais. L'organisation des filières animales va se modifier dans les années à venir en raison des déréglementations des marchés agricoles et de la montée des prix relatifs de l'énergie. Ces changements auront des effets ambigus sur la situation environnementale des bassins d'élevage. Ces changements peuvent, d'une part favoriser une meilleure valorisation des effluents d'élevage mais, d'autre part renforcer le processus d'agglomération des productions animales.

\section{Remerciements}

Je tiens à remercier Jean Boiffin, Vincent Chatellier, Pierre Dupraz, Sabine DuvaleixTréguer, Philippe Le Goffe, Jean-Louis Peyraud, Michel Rieu, Christine Roguet et Bertrand Schmitt avec qui j'ai eu de nombreuses discussions très stimulantes sur la situation et le devenir des filières animales. Evidemment les avis exprimés dans cet article sont ceux uniquement de l'auteur. Je remercie également Monique Harel et Sylvain Cariou pour leurs aides précieuses concernant les données ainsi que Sophie Le Perchec pour son travail sur la bibliographie, Catherine Donnars, les évaluateurs et la rédaction de la revue pour leurs relectures minutieuses de ce texte.

\section{Références}

Abdalla C.W., Lanyon L.E., Hallberg M.C., 1995. What we know about historical trends in firm location decisions and regional shifts: Policy issues for an industrializing animal sector. Am. J. Agric. Econ., 77, 1229-1236.

Aggarwal R.M., Lichtenberg E., 2005. Pigouvian taxation under double moral hazard. J. Environ. Econ. Manag., 49, 301-310.

Ambec S., Barla P., 2005. Quand la réglementation environnementale profite aux pollueurs : survol des fondements théoriques de l'hypothèse de Porter. Working paper, 20p.

Azzam A., Skinner C.S., 2007. Vertical economies and the structure of US Hog farms. Can. J. Agric. Econ., 55, 349-364.

Bagoulla C., Chevassus-Lozza E., Daniel K., Gaigné C., 2010. Regional production adjustment to import competition: Evidence from the French Agro-Industry. Am. J. Agric. Econ., 92, 1040-1050.

Ben Arfa N., Rodriguez C., Daniel K., 2009. Dynamiques spatiales de la production agricole en France. Rev. Econ. Rég. Urbaine, 4, 807-834.

Ben Arfa N., Daniel K., Jacquet F., 2010. A Generalized cross entropy approach to analyse the structural and spatial change in the French dairy farms. $4^{\text {emes }}$ Journ. Rech. Sci. Soc., INRASFER-CIRAD, 9-10 décembre, Rennes, France, $35 \mathrm{p}$.

Bushnell J.B., Mansur E.T., 2011. Vertical targeting and leakage in carbon policy. Am. Econ. Rev., 101, 263-267.

Butault J.P., 2006.La baisse des revenus et l'essoufflement de la productivité dans l'agriculture française depuis 1998. INRA, Sciences Sociales, $\mathrm{N}^{\circ} 2.8 \mathrm{p}$.

Canton J., Soubeyran A., Stahn H., 2008. Environmental taxation and vertical cournot oligopolies: How eco-industries matter. Environ. Res. Econ., 40, 369-382.
Chatellier V., Dupraz P., 2011. Politiques et dynamique des systèmes de production: Comment concilier compétitivité, défi alimentaire et environnement ? $6^{\text {eme }}$ Edition des Entretiens du Pradel, 15-16 septembre, Mirabel, France, 27p.

Chavas J.P., 2008. On the economics of agricultural production. Aust. J. Agric. Res. Econ., 52, 365-380.

Conseil des communautés européennes 1991. Directive 91/676/CEE du Conseil, du 12 décembre 1991, concernant la protection des eaux contre la pollution par les nitrates à partir de sources agricoles Journal officiel, $\mathrm{N}^{\circ} \mathrm{L} 375$, 31 décembre, 1-8.

Cronon W., 1991. Nature's metropolis: Chicago and the great west, New-York: Norton \& $\mathrm{Co}, 592 \mathrm{p}$.

Desrier, M., 2011, Les productions se concentrent dans les exploitations spécialisées. Agreste Primeur, №272.

Djaout F., Le Goffe P., Tauber M., 2009. Comment appliquer la directive nitrates et à quel coût ? Une modélisation spatiale du marché de l'épandage. Econ. Prév., 188, 43-61.

Dupraz P., 1997. La spécialisation des exploitations agricoles : changements techniques et prix des facteurs. Cah. Econ. Sociol. Rurales, 45, 94-122.

Dupraz P., Ruas J.F., Samson E., 2010. Le calcul d'indicateurs environnementaux selon l'analyse de cycle de vie à partir du RICA. Séminaire José Rey: Impact des mesures agroenvironnementales et des soutiens au développement rural. Montreuil sous Bois, France, 01 février, 47p.

Duvaleix-Tréguer S., Gaigné C., 2011. On the nature and the magnitude of scale economies in hog production. Mimeo.

Fujita M., Thisse J.F., 2002. Economics of agglomeration; cities, industrial location and regional growth. Cambridge University Press, Cambridge, UK, 466p.

Gaigné C., Le Gallo J., Larue S., Schmitt B., 2011. Does manure management regulation work against agglomeration economies? evidence from France. Am. J. Agric. Econ., 94, 116-132.

GCL Développement Durable 2010. État, perspectives et enjeux du marché des engrais : Ministère de l'Alimentation, de l'Agriculture et de la Pêche, Service de la Statistique et de la Prospective, 94p.

Gervais J.P., Bonroy O., Couture S., 2008. A province-level analysis of economies of scale in canadian food processing. Agribusiness, 24, 538-556.

Goodwin H.L., 2005. Location of production and consolidation in the processing industry: The case of poultry. J. Agric. Appl. Econ., 37, 339-346.

Herath D.P., Weersink A.J., Carpentier C.L., 2005. Spatial and temporal changes in the US Hog, dairy, and fed-cattle sectors, 1975-2000. Rev. Agric. Econ., 27, 49-69.

Hubbell B.J., Welsh R., 1998. An examination of trends in geographic concentration in U.S. hog production, 1974-96. J. Agric. Appl. Econ., 30, 285-299.

IFIP 2006. Le Porc par les Chiffres : édition 2006 Paris, France, ITP, 52p.

Ilari E., Daridan D., Desbois D., Fraysse J.L., Fraysse J., 2003. Les systèmes de production du porc en France : typologie des exploitations agricoles ayant des porcs. Paris, France, OFIVAL, 124p.

Isik M., 2004. Environmental regulation and the spatial structure of the US dairy sector. Am. J. Agric. Econ., 86, 949-962.

Kaplan J.D., Johansson R.C., Peters M. 2004. The manure hits the land: Economic and 
environmental implications when land application of nutrients is constrained. Am. J. Agric. Econ., 86, 688-700.

Krugman P., 1997. Development, geography, and economic theory. MIT Press Books, Massachusetts, USA, 127p.

Larue S., Abildtrup J., Schmitt B., 2011. Positive and negative agglomeration externalities: Arbitration in the pig sector. Spatial Econ. Analysis, 6, 167-183.

Le Goffe P., Salanie J., 2005. Le droit d'épandage a-t-il un prix ? Mesure sur le marché foncier. Cah. Econ. Sociol. Rurales, 77, 35-62.

Lelyon B., Chatellier V., Daniel K., 2012. Fin des quotas laitiers, contractualisation et stratégies productives: enseignements d'une modélisation bioéconomique. INRA Prod. Anim., 25, 67-76.

MacDonald J.M., Ollinger M.E., 2000. Scale economies and consolidation in hog slaughter. Am. J. Agric. Econ., 82, 334-346.

MacDonald J.M., McBride W.D., 2009. The transformation of U.S. livestock agriculture: scale, efficiency, and risks. Electronic Information Bulletin, 43, 46p.

Mansur E., 2011. Upstream versus downstream implementation of climate policy. In: Fullerton D., Wolfram C. (Eds). The design and implementation of U.S. climate policy. University of Chicago Press, in press.
Metcalf G.E., Weisbach D., 2009. The design of a carbon tax. Harvard Environ. Law Rev., 33, 499-556.

Metcalfe M., 2001. US hog production and the influence of state water quality regulation. Can. J. Agric. Econ., 49, 37-52.

Mosheim R., Lovell C.A.K., 2009. Scale economies and inefficiency of US dairy farms. Am. J. Agric. Econ., 91, 777-794.

Ollinger M., MacDonald J.M., Madison M., 2005. Technological change and economies of scale in U.S. poultry processing. Am. J. Agric. Econ., 87, 116-129.

Perrot, C., Chatellier, V., 2009. Evolution structurelle et économique des exploitations laitières du nord de l'Union européenne de 1990 à 2005 : des trajectoires contrastées. Fourrages, 197, 25-46.

Peyraud J.L., Cellier P., Donnars C., Réchauchère O., 2012. Les flux d'azote liés aux élevages, réduire les pertes, rétablir les équilibres. Expertise scientifique collective, INRA, France.

Porter M.E., van der Linde C., 1995. Toward a new conception of the environment-competitiveness relationship. J. Econ. Perspect., 9, 97-118.

Robreau F., Massabie P., Martin-Houssart G., 2010. Les bâtiments d'élevage porcin entre 2001 et 2008 : La gestion des effluents dans les élevages porcines. Agreste Primeur, 248, 4p.
Roe B., Irwin E.G., Sharp J.S., 2002. Pigs in space: Modeling the spatial structure of hog production intraditional and nontraditional production regions. Am. J. Agric. Econ., 84, 259-278.

Rosenthal S., Strange W., 2004. Evidence on the nature and sources of agglomeration economies. In: Henderson V., Thisse J.F. (Eds). Handbook of Regional and Urban Economics, Vol. 4, North Holland, 2119-2171.

Smith E.G., Card G., Young D.L., 2006. Effects of market and regulatory changes on livestock manure management in Southern Alberta. Can. J. Agric. Econ., 54, 199-213.

Tregaro Y., Lossouarn J., 2004. Dynamique de la production porcine mondiale et européenne : enjeux de localisation et de relations au territoire. Séminaire Porcherie Verte, 7-8 décembre, La Rochelle, France.

Weersink A., Eveland C., 2006. The siting of livestock facilities and environmental regulations. Can. J. Agric. Econ., 54, 159-173.

Wieck C., Heckelei T., 2007. Determinants, differentiation, and development of short-term marginal costs in dairy production: an empirical analysis for selected regions of the EU. Agric. Econ., 36, 203-220.

\section{Résumé}

Depuis plusieurs décennies, on assiste à un double mouvement de concentration industrielle et spatiale dans les filières animales en France, comme dans de nombreux pays d'Europe et d'Amérique du Nord. La baisse du prix relatif de l'énergie, la présence d'économies d'échelle aux différents stades des filières, les gains économiques liés à la proximité géographique entre les éleveurs et les industries amont et aval constituent des facteurs importants de ce processus de concentration spatiale des productions animales. Cette agglomération se traduit cependant par une concentration des effluents d'élevage, dont l'épandage a des conséquences environnementales souvent néfastes. Des mesures de régulation environnementale ont vu le jour, notamment au travers de la Directive Nitrates de 1991. La question de la localisation des productions agricoles et notamment de sa dispersion a été évoquée comme un levier possible pour réduire les problèmes environnementaux, mais il apparaît que les mesures actuelles sont insuffisantes pour contrecarrer les facteurs d'agglomération à l'origine des excès d'azote dans les territoires spécialisés dans les productions animales. Plusieurs arguments peuvent en revanche être avancés montrant qu'il pourrait être plus efficace d'appliquer des régulations au niveau des filières plutôt que seulement sur les éleveurs.

\section{Abstract}

\section{Organisation of the livestock sector and the environment. 20 years after the nitrate directive}

During the last decades, a double movement of industrial and spatial concentration has been observed in the livestock sector in France, as in many countries in Europe and North America. The falling of the relative price of energy over this period, the presence of scale economies at different stages of animal industries, the gains associated with the spatial concentration and geographical proximity between farmers and downstream/upstream industries are key factors explaining the agglomeration of animal production. However, this spatial concentration of animal production is a serious source of watercourse pollution worldwide. Different measures of environmental regulation have emerged, notably through the 1991 Nitrate Directive, but the current policies are insufficient to counteract these agglomeration factors causing excess nitrogen in the territories specialized in livestock production. Several arguments can be advanced showing that environmental regulation could be more effective if it concerned not only farmers but also downstream/upstream industries.

GAIGNÉ C., 2012. Organisation des filières animales et environnement. Vingt ans après la directive nitrates. INRA Prod. Anim., 25, 375-388. 